\title{
JOGOS DE EMPRESAS E MÉTODO DO CASO: CONTRIBUIÇÕES AO PROCESSO DE ENSINO E APRENDIZAGEM EM ADMINISTRAÇÃO
}

\author{
BUSINESS GAMES AND THE CASE METHOD: \\ CONTRIBUTIONS TO THE PROCESS OF TEACHING \\ AND LEARNING IN MANAGEMENT
}

Recebido em: $18 / 02 / 2013$ A Aprovado em: 26/04/2013
Avaliado pelo sistema double blind review
Editora Científica: Manolita Correia Lima

\author{
SHEILASERAFIM DASILVA sheila_serafim@yahoo.com.br \\ MURILO ALVARENGA OLIVEIRA \\ GUSTAVO DA SILVA MOTTA \\ UNIVERSIDADE FEDERAL FLUMINENSE
}

\section{RESUMO}

Este estudo analisou a aplicação conjunta das técnicas jogos de empresas e método do caso em comparação à aplicação exclusiva de jogos de empresas. São apresentados no estudo os fundamentos dos jogos de empresas, aprendizagem vivencial e método do caso no campo da Problem-Based Learning (PBL) que se desloca das abordagens convencionais de ensino, centrada no papel do professor, para uma abordagem centrada no aluno. O estudo caracteriza-se como uma pesquisa de natureza aplicada, realizada por meio de um experimento controlado com duas turmas de um curso de administração numa Universidade Pública. O grupo de teste recebeu as orientações das regras do simulador dinamizadas pelos fundamentos do método do caso ao contrário do grupo de controle que recebeu as instruções de forma expositiva. Os dados foram coletados com a aplicação de dois testes de conteúdo e com os resultados do jogo de empresas. A análise ocorreu de forma quantitativa com tratamento estatístico usando teste de hipótese. Os resultados preliminares da pesquisa mostraram que não houve diferenças significativas na assimilação do conhecimento de ambas as turmas. Entre as contribuições do estudo destacam-se as reflexões sobre o processo de ensino e aprendizagem em administração por meio de experimentos controlados.

Palavras-chave: jogos de empresas; método do caso; aprendizagem em administração.

\begin{abstract}
This study examined the joint application of business games and the case method, compared to the application of only business games. The paper presents the fundamentals of business games, experiential learning and the case method approach within the field of Problem-Based Learning $(P B L)$ which moves from conventional teaching approaches, which focus on the role of the teacher, to a constructivist approach, which is student-centered. The study is of an applied research nature, conducted through a controlled experiment with two groups from a management course at a public university. The test group received orientation on the simulator rules utilizing the case method approach, while the control group received instructions using the expository method. Two content tests and the results of the business game were used to gather the data, with quantitative analysis and hypothesis testing subsequently employed. The preliminary results of the survey show that there were no significant differences in the assimilation of knowledge between the groups. The use of controlled experiments to assist thinking about the teaching and learning process within management courses stood out among the study's contributions.

Keywords: business games; case method; learning in management.
\end{abstract}




\section{INTRODUÇÃO}

Segundo dados do Instituto Nacional de Estudos e Pesquisas Educacionais Anísio Teixeira (INEP, 2009), havia 874.076 alunos matriculados em cursos de graduação presenciais de administração em todo o Brasil, no ano de 2009. Esse dado corresponde a $17, \mathrm{I} \%$ do total de alunos matriculados nos cursos de graduação presenciais do país. Adicionando a expansão de cursos de graduação não presenciais, além da pós-graduação (stricto sensu e lato sensu) e da educação executiva. Observa-se que a área de administração assumiu uma dimensão maior do que qualquer outra em nosso país, como observado também por Bertero (2006).

Essa expansão de cursos de administração, não necessariamente está associada à melhoria dos processos de ensino e aprendizagem. Projetos como o PCDA (Programa de Capacitação Docente em Administração) da AnPad (Associação Nacional de Pós-graduação e Pesquisa em Administração) e o Pró-Administração da CAPEs (Coordenação de Aperfeiçoamento de Pessoal de Nível Superior), evidenciam a preocupação de professores e pesquisadores da área em relação aos aspectos contemporâneos de ensino e aprendizagem em administração.

A maior preocupação é se a qualidade desses processos está crescendo no mesmo ritmo que a demanda, ou até mesmo que a oferta, e contribuindo para a formação de executivos que irão gerenciar grandes empresas. Muito tem se questionado acerca do ensino em administração e suas metodologias. De um lado o ensino teórico e reprodutor dos modelos de gestão existentes; de outro uma profissão que exige desempenho extremamente prático e técnico, onde não só o conhecimento teórico basta, mas também um conjunto de competências de caráter interpessoais. (BENNIS; O'TOOLE, 2005; BERTERO, 2003; MINTZBERG; GOSLING, 2003; NICOLINI, 2003; PFEFFER; FONG, 2003; TUSHMAN et al., 2007).

Bennis e O’Toole (2005, p. 62) afirmam que "a administração é uma profissão, assim como a medicina e o direito, e escolas de gestão são escolas profissionais - ou deveriam ser". O que se ensina nesses cursos precisa estar relacionado com a prática da profissão de administrador. 
Ou estariam as universidades focadas em uma pesquisa não aplicada e em um ensino com ausência de exemplos práticos ou experiências? A administração é uma ciência aplicada, assim, tanto o ensino quanto a pesquisa na área exigem uma relação com o exercício da profissão e com os problemas gerenciais enfrentados por administradores em atuação.

A formação de administradores deve integrar conhecimento e prática assim como o exercício da profissão. Entretanto, observa-se que as escolas de administração estão cada vez mais desatreladas da prática e da pesquisa aplicada carecendo de uma relevância no mundo real e dessa forma, a lacuna entre uma pesquisa científica de qualidade (rigor) e um ensino com experiências práticas (relevância) afeta a qualidade do ensino, bem como a legitimidade das escolas de negócios (BENNIS; o'toole, 2005; Mintzberg, 2004; tushman et al., 2007). Paradoxalmente, como observam Bordenave e Pereira (2006) há um emprego excessivo, quase exclusivo, de aulas expositivas e estas não atendem a necessidade real das organizações.

Neste contexto, muitos são os desafios que se apresentam em relação ao ensino de administração. De que forma o conhecimento pode ser transmitido e ao mesmo tempo assimilado? Por meio de inúmeras aulas expositivas ou por meio da participação ativa dos alunos em análise de casos, vivência, solução de problemas e troca de experiências? Um dos grandes desafios das escolas de administração é saber quais os métodos ou técnicas podem ser utilizados para auxiliar um aprendizado mais significativo em gestão (BORDENAVE; PEREIRA, 2006; PFEFFER; FONG, 2003).

Uma aprendizagem significativa compreende o envolvimento do aluno no seu próprio processo de aprendizagem. Bordenave e Pereira (2006, p. 47) sugerem que "o aluno, sob orientação do professor, deve ser parcialmente responsável pela solução do problema ou pela descoberta de meios que levem ao objetivo, e dessa forma, o que ele aprende é parte do que ele mesmo ajudou a criar". Os mesmos autores questionam a falta de uma "correspondência perfeita entre o que o professor ensina e o que o aluno aprende. Por que o ensino é tão pouco eficiente em termos esforço docente / aproveitamento discente?". 
Katz (1974) desenvolveu um modelo, composto por três tipos de habilidades necessárias para que o administrador realize seu trabalho eficazmente: as técnicas; as humanas; e as conceituais. Para o autor, tais habilidades podem e devem ser desenvolvidas. Assim, Katz (1974) defende que o processo de formação de administradores deve visar à ação efetiva deste profissional em situações diversas. No âmbito do curso de administração, conseguir abordar toda a complexidade organizacional, dadas as muitas relações existentes entre as diversas disciplinas, é talvez o maior dos desafios para o ensino de administração. As disciplinas, devido à própria divisão do curso em frações curriculares isoladas, podem não ser capazes de dar conta das suas inter-relações.

Algumas experiências têm permitido, há algum tempo, especificamente nos cursos de administração, a introdução de técnicas que enfocam tais desafios. Exemplos dessas técnicas são as simulações por meio de jogos de empresas (DICKINSON; FARIA, I994; KEYS; WOLFE, I990; ROSAS; SAUAIA, 2006) e O método do caso (BARNES; CHRISTENSEN; HANSEN, I994; IKEDA; VELUDO-DEOLIVEIRA; CAMPOMAR, 2005; ROESCH; FERNANDES, 2006; MENEZES, 2009). 


\section{PROBLEMA DE PESQUISA E OBJETIVOS}

A escolha de se trabalhar com as referidas técnicas deve-se a busca por fugir de um modelo convencional, no qual o aluno tem o papel passivo de assistir à exposição do professor. A aplicação de jogos de empresas e do método do caso permite ao aluno buscar e manipular informações de acordo com os seus próprios interesses e percepções, assumindo o papel ativo no processo de ensino e aprendizagem e a responsabilidade pela construção do seu próprio conhecimento.

Tem-se, entretanto, que cada uma das técnicas (jogos de empresas e método do caso) apresenta alcances e limites específicos e vem evoluindo separadamente, embora ambas pertençam à aprendizagem vivencial (NAKANO; TERANO, 2005). Com o método do caso, estimula-se a busca pelo conhecimento necessário à solução do problema proposto, mas não é possível testar as decisões dos alunos (NAKANO; MATSUYAMA; TERANO, 2007; SAUAIA, 2006; TOMPSON; DASS, 2008). Já com os jogos de empresas é oferecido o feedback das decisões de forma quantitativa, entretanto, observa-se um foco maior no desenvolvimento de atitude gerencial e baixa capacidade de aquisição de novos conhecimentos (sanTos, 2003). Suspeita-se, desta forma, da complementaridade entre as técnicas jogos de empresas e método do caso, já que as limitações de uma são atendidas pela outra, o que leva à seguinte investigação: quais os limites e possibilidades da aplicação conjunta do método do caso com jogos de empresa no processo de ensino e aprendizagem em administração?

Este estudo pretende identificar e analisar os limites e possibilidades da aplicação conjunta das técnicas jogos de empresas e método do caso em comparação à aplicação exclusiva de jogos de empresas. Desta forma, espera-se contribuir para o avanço qualitativo dos processos de ensino e aprendizagem em administração, apelando não apenas ao aspecto lúdico, mas principalmente, enfocando o desenvolvimento de habilidades e conhecimentos necessários ao profissional administrador.

De forma específica, busca-se medir se a aplicação conjunta de jogos de empresas e do método do caso em comparação à aplicação exclusiva 
de jogos de empresas, com base na percepção do aprendiz, permite: (I) Maior aquisição de conhecimento; (2) Maior familiaridade com o ambiente de negócios; (3) Maior desenvolvimento do ponto de vista da alta administração; (4) Vislumbrar melhor as tarefas das áreas funcionais; (5) Melhor desenvolvimento das habilidades para a aplicação correta dos conceitos e técnicas administrativas; (6) Melhor desenvolvimento da capacidade de integrar as habilidades necessárias ao sucesso do trabalho em equipe.

Neste estudo, após esta introdução, serão apresentadas as características do método do caso no campo da Aprendizagem Baseada em Problemas (PBL), e num segundo momento, as características dos jogos de empresas no contexto da Aprendizagem Vivencial. A seguir, serão expostas as vantagens da aplicação das técnicas em conjunto e sua contribuição para o ensino e aprendizagem em administração. 
FUNDAMENTAÇÃO TEÓRICA

\section{CARACTERÍSTICAS DO MÉTODO DO CASO}

A fim de evitar qualquer conflito conceitual entre os termos estudo de caso e método do caso, faz-se necessário tal distinção: o estudo de caso é uma técnica de pesquisa utilizada no processo de investigação, enquanto o método do caso é um recurso didático para aplicação de teorias em salas de aula com o uso de casos. Sendo um caso, no campo da administração, a descrição de uma situação ou problema organizacional com base em acontecimentos reais.

O método do caso foi introduzido na Harvard Business School em 1908, em cursos de Direito Comercial, sendo algumas aulas, atualmente, ministradas quase exclusivamente, por meio da discussão de casos (ROESCH; FERNANDES, 2006). O método consiste em uma estratégia educacional cujo intuito é levar os estudantes a refletirem sobre situações apresentadas no caso e envolvê-los na tomada de decisões sobre o episódio estudado. Ao trazer a realidade do ambiente empresarial para dentro da sala de aula, aproxima os alunos das práticas de gestão.

O uso de casos faz com que os participantes analisem e reflitam situações diversas de forma dinâmica, participativa e criativa propiciando o desenvolvimento do raciocínio lógico e o pensamento crítico. No processo, o professor assume o papel de instrutor ao invés de dar as respostas prontas, dessa forma, o aluno torna-se responsável por parte do seu processo de aprendizagem como proposto por Bordenave e Pereira (2006).

O fator crítico ao sucesso do caso como um recurso pedagógico repousa enormemente no uso que se faz dele. Para Oliveira (2000) é um engano tanto o uso expositivo do caso em sala de aula quanto o seu uso pontual, pelo docente, para a demonstração de uma teoria. As duas formas colocam o professor no centro do processo, enquanto o verdadeiro potencial de aprendizagem estaria no enfoque no aprendiz; à medida que o mesmo enfrenta as dúvidas conceituais e manipula os dados, é capaz de conferirlhes sentido próprio. 
Para Li e Baillie (1993), o método do caso tem grande valor para a educação gerencial por refletir uma experiência real e criar uma situação de aprendizagem que requer pensamento crítico e análise. Entretanto, sua principal limitação está na incapacidade de os alunos testarem suas decisões.

\section{MÉTODO DO CASO NO CONTEXTO DA APRENDIZAGEM BASEADA EM PROBLEMAS (PBL)}

A Aprendizagem Baseada em Problemas (Problem-Based Learning - PBL), assim como o método do caso, propõe o desenvolvimento de habilidades gerenciais por meio da busca por solução de problemas organizacionais. Dessa forma, o método do caso está inserido na metodologia de ensino proposta pela $\mathrm{PBL}$, atendendo aos critérios da aprendizagem centrada no aluno com produção de conhecimento integrado, contextualizado e cooperativo.

A PBL é um método de ensino e aprendizagem que consiste na discussão e solução de problemas com foco no papel do estudante, tornando-o um elemento ativo no processo e um aprendiz por toda a vida. O método propõe uma aprendizagem mais eficaz por meio do desenvolvimento da comunicação, trabalho em equipe, postura crítica e foco na solução de problemas. A aplicação de tal metodologia possui uma série de vantagens, entre elas: (I) forte motivação do aluno para o aprendizado; (2) desenvolvimento de raciocínio analítico e crítico e (3) desenvolvimento de habilidades de autoaprendizado.

Entretanto, a PBL apresenta algumas limitações para os alunos, entre elas, destaca-se que, em algumas práticas, como nos jogos de empresas, por exemplo, pode ser desnecessário o conhecimento das teorias mais avançadas e, ao mesmo tempo, o conhecimento memorizado pode ser insuficiente. Além disso, a metodologia impõe ao indivíduo o ritmo de atividade da equipe, o que pode ser frustrante para alunos que possuem dificuldades para produzir dessa maneira. Segundo os autores, outra desvantagem do método é a impossibilidade de se trabalhar todos os conceitos de uma disciplina. 


\section{CARACTERÍSTICAS DOS JOGOS DE EMPRESAS}

Os jogos de empresas têm sido utilizados desde a década de 50 na Universidade de Washington, e desde 1970, em instituições brasileiras (Keys; WOlFe, I990; Oliveira; SAUAia, 2009). Atualmente, inúmeras são as faculdades de administração que contam, em seu currículo, com um componente denominado "Jogos de Empresas".

Os jogos de empresas são reconhecidos como um método dinâmico de ensino onde os participantes ao analisar cenários se envolvem em um processo de tomada de decisão e recebem o feedback da simulação. Rosas e Sauaia (2009) define-os como vivências de gestão, na qual participantes elaboram estratégias e se envolvem em um processo de tomada de decisão. Para aplicação do jogo faz-se necessário o uso de um simulador, que pode ser informatizado ou não. $\mathrm{O}$ simulador organizacional trata-se da ferramenta utilizada para propiciar a tomada de decisão e gerar relatórios - feedback aos participantes.

Outros autores (KEYS; WOLFE, I990; PRETTO; ALMEIDA, 2007; STAHL; LOPES, 2004) apresentam alguns benefícios na aplicação do método: (I) permite aos participantes identificar as consequências progressivas de suas decisões, desenvolvendo uma visão de longo prazo; (2) maior grau de realismo proporcionado por uma vivência que aproxima o participante da realidade empírica onde irá atuar profissionalmente; (3) possibilidade de integração das disciplinas curriculares, proporcionando uma visão sistêmica de uma organização; (4) maior envolvimento e participação do aluno na atividade; (5) proporciona um feedback das decisões.

Com os jogos é possível visualizar o resultado das decisões e modificálas em diferentes períodos de tempo simulados. Esta diferença ilustra as principais vantagens de utilização dos jogos de empresas no processo de ensino e aprendizagem em gestão: a capacidade de observar as consequências de suas decisões e a possibilidade de se aprender com os erros. No entanto, Sauaia (2006) afirma que o ensino, por meio de jogos de empresas, não auxilia a obtenção de conhecimento teórico e aplicado. É nesse sentido que Arbex (2005) alerta para o fato de que os jogos de empresas não devem ser utilizados como substitutos a outras técnicas e metodologias educacionais. 
Deve-se pensar em seu uso como complementar e auxiliar ao processo.

Os jogos de empresas revelam competências não captadas pelos métodos de ensino tradicionais, conforme Sauaia (2010). Esta discussão abre espaço para a aprendizagem vivencial, abordagem altamente aderida ao desenvolvimento cognitivo por meio dos jogos de empresas.

\section{APRENDIZAGEM VIVENCIAL E OS JOGOS DE EMPRESAS}

Segundo Kolb (1984), a aprendizagem vivencial considera o aprendizado como um processo, no qual o conhecimento é produzido por meio da transformação da experiência. Desta forma, é possível extrair três aspectos fundamentais da aprendizagem vivencial. Inicialmente, observa-se uma ênfase no processo e na aprendizagem, em oposição à ênfase no conteúdo e no resultado, característicos do ensino tradicional. Segundo, o conhecimento é visto como um processo de transformação que é continuamente criado e recriado, ao invés de um produto acabado que deve ser adquirido ou transmitido. Finalmente, a aprendizagem vivencial reconhece que os indivíduos aprendem de diferentes formas e respondem distintamente a um mesmo estímulo (коLв, 1984).

Para Kolb (1984), há quatro capacidades básicas que o aprendiz precisa dispor para efetivar seu aprendizado. Tais capacidades se organizam em quatro fases que serão descritas a seguir e que compõem o Ciclo da Aprendizagem Vivencial ou Ciclo de Kolb:

A Experiência Concreta que é a orientação, cujo enfoque reside no envolvimento com experiências e no tratamento de questões humanas. Enfatiza o sentir em oposição ao pensar e interessa-se por questões particulares ao invés de generalizações.

B Observação Reflexiva, cujo fim é a compreensão, por meio da observação e descrição cuidadosa e imparcial, do significado das ideias e dos fatos. Privilegia refletir em oposição ao agir e interessase na verdade absoluta ou em como as coisas são ao invés do seu funcionamento. 
C Conceituação Abstrata, cujo enfoque está no uso da lógica, das ideias e dos conceitos. Privilegia o pensar em oposição ao sentir e interessa-se por teorias gerais ao invés de questões particulares.

D Experimentação Ativa que é a orientação onde o foco está em influenciar ativamente as pessoas e mudar as situações. Enfatiza o agir em oposição ao refletir e interessa-se pragmaticamente no que deve ser feito ao invés da busca pela verdade absoluta.

Sauaia (2008) sugere a aplicação dos jogos de empresas, obedecendo às fases do Ciclo de Kolb (1984). Desta forma, as regras econômicas do simulador e a materialização da experiência por meio da tomada de decisão inicial representariam a experiência concreta. A observação reflexiva seria representada pela análise dos resultados e a observação dos desvios entre as metas planejadas e realizadas. Uma sessão de debates sobre os diversos desempenhos da turma pode levar à compreensão dos resultados, com base na revisão conceitual dos modelos teóricos, o que se caracterizaria como a fase de Conceituação Abstrata. E, completando o ciclo, a experimentação ativa seria representada pela adaptação do rumo das decisões, face aos resultados obtidos. 


\section{COMBINAÇÃO ENTRE JOGOS DE EMPRESAS E MÉTODO DO CASO}

Há poucos estudos (MOTTA; ARMOND-DE-MELO, 2008; NAKANO; TERANO, 2004; SAUAIA, 2006; TOMPSON; DASS, 2000) que tentam integrar casos e jogos de empresas. No entanto, as duas abordagens vêm evoluindo separadamente e cada uma possui suas vantagens próprias (NAKANO; MATSUYAMA; TERANO, 2007).

A análise das características do método do caso e dos jogos de empresas mostra uma revisão nas práticas de ensino, atendendo às necessidades mais contemporâneas, dada a configuração hodierna das turmas que compõem os cursos de administração. O Quadro I apresenta uma síntese dessas características.

Quadro I Características do método do caso e dos jogos de empresas

\begin{tabular}{|lll|}
\hline Característica & Mécnica de Ensino & Jogos de Empresas \\
\hline Papel do Estudante & Autonomia no processo & Autonomia no processo \\
\hline Papel do Professor & Desafiar e interagir & Auxiliar o Processo \\
\hline Objetivo Educacional & Conhecimento e discussão teórica & $\begin{array}{l}\text { Comportamento e Atitude } \\
\text { Gerencial }\end{array}$ \\
\hline Qualificação do problema & $\begin{array}{l}\text { Busca de conhecimento e articulação } \\
\text { de conceitos }\end{array}$ & $\begin{array}{l}\text { Estimula o envolvimento do } \\
\text { aluno }\end{array}$ \\
\hline Pontos Fortes & $\begin{array}{l}\text { Baixo Custo. Não necessita de recursos } \\
\text { de Tl ou infraestrutura específica. }\end{array}$ & $\begin{array}{l}\text { Maior interação pessoal entre } \\
\text { os alunos por meio da prática } \\
\text { vivencial. }\end{array}$ \\
\hline Pontos Fracos & Roteiro linear de solução de problemas & $\begin{array}{l}\text { Requer simuladores, o que } \\
\text { geralmente envolve altos custos. } \\
\text { Dificuldade para avaliação da }\end{array}$ \\
\hline Oprendizagem.
\end{tabular}




\begin{tabular}{|lll|}
\multicolumn{1}{|c}{ Técnica de Ensino } & Método do Caso & Jogos de Empresas \\
Caracteristica & & Humana \\
\hline $\begin{array}{l}\text { Principal } \\
\text { Competência desenvolvida }\end{array}$ & Conceitual e Técnica & \\
\hline
\end{tabular}

Fonte: Adaptado de Motta e Armond-de-Melo (2008).

A aplicação das técnicas proporciona uma mudança nos papéis do processo de ensino e aprendizagem. Enquanto o professor assume o papel de auxiliar ou facilitador, interagindo com o aluno e ao mesmo tempo desafiando-o na solução de problemas gerenciais, o aluno por meio da investigação encontra diferentes respostas para tais problemas. Dessa forma, o estudante tem autonomia no processo de ensino e aprendizagem, tornando-se responsável pela produção do seu próprio conhecimento.

A proposta de aplicação das técnicas de ensino tratadas neste estudo não tem como objetivo substituir as aulas conhecidas como expositivas, mas sim complementá-las. O método do caso, assim como a aula expositiva, proporciona aquisição de teorias e conceitos e discussão teórica, sendo neste momento, atendida uma restrição dos jogos de empresas.

Enquanto o método do caso enfoca o desenvolvimento do conhecimento, o jogo de empresas estimula comportamentos e atitudes gerenciais. Em ambos os casos têm-se objetivos que estão intrinsecamente ligados às habilidades/competências requeridas do profissional administrador.

De acordo com Motta e Armond-de-Melo (2008), no método do caso a principal competência desenvolvida é a conceitual e a técnica. A competência conceitual se deve ao desenvolvimento de uma visão sistêmica da organização. $\mathrm{O}$ aluno passa a visualizar todos os departamentos da empresa e suas relações, ao invés de áreas isoladas. Assim, os objetivos dos indivíduos estão atrelados aos da organização como um todo. A competência técnica, segundo os autores, consiste em utilizar conhecimentos, métodos, técnicas e equipamentos necessários para executar tarefas específicas, por meio da experiência e educação. Porém, o método do caso, em suas limitações, além de estar baseado simplesmente em texto impresso e seu cenário permanecer estático, não proporciona um feedback aos participantes. 
No caso dos jogos de empresas, segundo Motta e Armond-de-Mello (2008), a competência mais importante é a humana, que compreende o desenvolvimento das habilidades comportamentais, que inclui relacionamentos interpessoais, comunicação, iniciativa, liderança, capacidade para compreender, liderar e motivar pessoas. De acordo com Sauaia (2006) a grande vantagem de uma simulação é que ela oferece mais dramaticamente a sensação de encerramento e conquista que o método do caso carece.

Quanto à qualificação do problema, os jogos de empresas proporcionam maior envolvimento e interação das equipes, em contrapartida, estes não auxiliam a aquisição de conhecimento teórico, uma vez que seu foco está nas regras do simulador. Enquanto no método do caso busca-se mais conhecimento e articulação de conceitos e teorias.

Os aspectos apresentados demonstram que as técnicas jogos de empresas e método do caso, sozinhas, são limitadas em relação aos objetivos educacionais em administração.

Embora o método do caso seja amplamente difundido e o jogo de empresas tenha grande aceitação por parte das instituições de ensino, há outras dificuldades para adoção de tais métodos em sala de aula, entre eles, pode-se citar, no método do caso, a dificuldade na elaboração de casos ou ausência de experiência na condução dos mesmos (ROESCH; FERNANDES, 2006); e no caso dos jogos de empresas, pode-se citar o alto custo dos simuladores e a infraestrutura exigida para sua aplicação (KEYs; WOLFE, I990; SAUAIA, 2006; SANTOS; LOVATO, 2007).

Entretanto, há certa exigência por parte dos alunos que requerem práticas docentes inovadoras e diferenciadas. Como acompanhar as transformações do mundo ao redor das instituições que preparam os futuros gestores para atuar nele sem revolucionar suas técnicas de ensino e aprendizagem? Tais dificuldades para aplicação de novos métodos de ensino que atendam a necessidade das transformações devem ser superadas pelo corpo docente e pelas instituições.

As carências de um método de ensino devem ser supridas por outras metodologias a fim de obter uma maximização do aprendizado, sendo um 
único instrumento incapaz de criar todas as condições necessárias para um dado objetivo de aprendizagem.

Embora as referidas técnicas ofereçam diferentes experiências de aprendizagem e dão ênfase a diferentes habilidades, são similares quanto a sua finalidade. Neste sentido, sugere-se a aplicação das técnicas - jogos de empresas e método do caso - em conjunto, dada a sua complementaridade. Essa combinação busca reunir as vantagens e reduzir as limitações de ambos os métodos, uma vez que as limitações de uma são atendidas pela outra. E assim, desenvolver habilidades e atitudes gerenciais com o jogo de empresas e ao mesmo tempo criar uma discussão teórica baseada em problemas, com o uso do método do caso. 


\section{MÉTODO DE PESQUISA}

O experimento foi realizado no primeiro semestre de 20II, com duas turmas da disciplina Laboratório de Gestão Simulada I, inserida no terceiro período do curso de graduação em administração numa Instituição de Ensino Superior. Para um grupo foi aplicada a técnica de jogos de empresas combinada com o método do caso (grupo de teste), sem adoção de aulas expositivas para apresentação do caso empresarial e das regras do simulador. Este foi o grupo de teste, no qual a variável método do caso foi inserida como forma de se experimentar os resultados, de acordo com as seis dimensões do modelo teórico.

Os demais estudantes formaram o grupo de controle, que foi exposto à apresentação expositiva do caso empresarial e das regras do simulador e posteriormente, foi submetido exclusivamente, à técnica de jogos de empresas. Para se evitar a influência de demais variáveis extrínsecas, em ambos os casos o mesmo professor guiou o experimento, seguindo o mesmo roteiro e adotando o mesmo simulador empresarial. Durante o experimento adotou-se os procedimentos expostos no Quadro 2.

Quadro 2 Fases da Aplicação do Experimento

\begin{tabular}{|c|c|c|}
\hline Etapas & Turma A1 (Controle) & Turma A2 (Teste) \\
\hline $\begin{array}{l}\text { Apresentação das atividades } \\
\text { que seriam desenvolvidas ao } \\
\text { longo do semestre }\end{array}$ & $\begin{array}{l}\text { Exposição por parte do pro- } \\
\text { fessor }\end{array}$ & - Exposição por parte do professor \\
\hline $\begin{array}{l}\text { Apresentação do Caso } \\
\text { Empresarial e das Regras do } \\
\text { Simulador }\end{array}$ & $\begin{array}{l}\text { Método adotado - Aulas } \\
\text { expositivas }\end{array}$ & $\begin{array}{l}\text { Método adotado - Jogo de } \\
\text { Empresas e Método do Caso }\end{array}$ \\
\hline $\begin{array}{l}\text { Teste } 1 \text { - Assimilação de } \\
\text { conteúdo e aplicação de teoria }\end{array}$ & Teste 1 & Teste 1 \\
\hline $\begin{array}{l}\text { Rodada Experimental da } \\
\text { Simulação }\end{array}$ & Rodada Experimental & Rodada Experimental \\
\hline $\begin{array}{l}\text { Execução da técnica de } \\
\text { aprendizagem }\end{array}$ & $\begin{array}{l}\text { Exclusivamente Jogo de } \\
\text { Empresas }\end{array}$ & $\begin{array}{l}\text { Combinação entre Jogo de } \\
\text { Empresas e Método do Caso }\end{array}$ \\
\hline $\begin{array}{l}\text { Simulação (Rodadas do Jogo de } \\
\text { Empresas) }\end{array}$ & $\begin{array}{l}\text { Rodadas de } 1 \text { a } 4 \text { apoiando- } \\
\text {-se nas regras do simulador } \\
\text { (expostas pelo professor e } \\
\text { disponíveis no livro-texto) }\end{array}$ & $\begin{array}{l}\text { - Rodadas de } 1 \text { a } 4 \text { apoiando-se } \\
\text { nas regras do simulador. Porém, } \\
\text { transformadas em um caso } \\
\text { empresarial }\end{array}$ \\
\hline
\end{tabular}




\begin{tabular}{|llc|}
\hline Etapas & \begin{tabular}{l} 
Turma A1 (Controle) \\
\hline $\begin{array}{l}\text { Exercícios dirigidos durante a } \\
\text { simulação }\end{array}$
\end{tabular} & $\begin{array}{l}\text { Exercícios divididos por área Pequenos casos divididos por } \\
\text { funcional (Produção, RH, área funcional } \\
\text { Finanças, etc.) }\end{array}$ \\
\hline $\begin{array}{l}\text { Teste 2 - Assimilação de } \\
\text { conteúdo e aplicação de teoria }\end{array}$ & $\begin{array}{l}\text { Teste } 2 \\
\text { Análise e Verificação das } \\
\text { hipóteses }\end{array}$ & $\begin{array}{l}\text { Verificação das hipóteses por meio de teste de diferença de } \\
\text { média em relação aos testes de assimilação de conhecimento e os } \\
\text { resultados no Jogo de Empresas }\end{array}$ \\
\hline
\end{tabular}

Como instrumentos de medição da aprendizagem foram aplicados dois testes em momentos distintos para verificar a assimilação conceitual e a aprendizagem. O primeiro teste continha questões de assimilação de conteúdos teóricos e o segundo, aplicado após a simulação, continha questões de aplicação prática de conteúdos.

Os dados coletados com a aplicação dos testes de conteúdo receberam um tratamento estatístico como o uso de um teste de hipótese que busca identificar a relação existente entre as variáveis analisadas. Para isto, definiram-se a hipótese nula $\left(\mathrm{H}_{\mathrm{o}}\right)$ e alternativa $\left(\mathrm{H}_{\mathrm{r}}\right)$. O teste " $\mathrm{F}$ " de comparação de variâncias foi utilizado com o intuito de verificar se as variâncias das duas turmas (teste e controle) são iguais como um procedimento preliminar no teste de comparação de médias. Em seguida, com o auxílio do teste "F", definiu-se a utilização do teste " $t$ " de comparação de médias presumindo variâncias equivalentes. 


\section{ANÁLISE DESCRITIVA DO EXPERIMENTO}

As hipóteses consideradas para aplicação dos testes estatísticos foram: (a) hipótese nula $\left(\mathrm{H}_{\mathrm{o}}\right)$ - o quadro teórico apresentado da turma 2 (grupo de teste) não apresenta perda ou ganho na absorção de conhecimento em relação à turma I (grupo de controle), mesmo sendo expostas a duas abordagens diferentes; (b) hipótese alternativa $\left(\mathrm{H}_{\mathrm{r}}\right)$ - considera um ganho ou perda na assimilação do conteúdo considerada relevante estatisticamente.

A primeira etapa compreende a busca por diferenças significativas nos resultados do teste I (conteúdo teórico) aplicado para o grupo de controle (turma I) em relação aos resultados do mesmo teste aplicado para o grupo de teste (turma 2). A princípio, aplicou-se o teste "F" de comparação de variâncias, conforme dito anteriormente. Em seguida, o mesmo procedimento foi realizado, porém com os resultados do teste 2 (conteúdo prático). O resultado pode ser observado na Tabela I.

Tabela I Teste-F: duas amostras para variâncias

\begin{tabular}{|lllll|} 
& Teste 1 & \multicolumn{3}{c|}{ Teste 2 } \\
& Turma 1 (Controle) & Turma 2 (Teste) & Turma 1 (Controle) & Turma 2 (Teste) \\
\hline Média & 5,642361 & 5,6974 & 5,25 & 5,170213 \\
\hline Variância & 1,137184 & 1,710522 & 2,193548 & 2,883441 \\
\hline Observaçôes & 32 & 47 & 32 & 47 \\
\hline GI & 31 & 46 & 31 & 46 \\
\hline F & 0,664817 & & 0,76074 & \\
\hline P(F<=f) uni-caudal & 0,11649 & & 0,212782 & \\
\hline F crítico uni-caudal & 0,568569 & & 0,568569 & \\
\hline
\end{tabular}

Observa-se que os pontos percentuais de diferença entre as duas turmas nos dois testes de conteúdo (teórico e prático) não são significativos e as variâncias podem ser consideradas equivalentes. Desta forma, definiuse a aplicação do teste " $\mathrm{t}$ " para duas amostras presumindo variâncias equivalentes (Tabela 2). A distribuição " $\mathrm{t}$ ” de student é usada quando se tem amostras pequenas $(n<30)$, a população é normalmente distribuída 
e $\sigma$ é desconhecido. Atendendo a estas exigências o teste " $t$ " foi utilizado a fim de identificar diferenças relevantes entre os efeitos percebidos pelos sujeitos da pesquisa, conforme os dados apresentados. O teste $t$ é um teste paramétrico que utiliza duas amostras independentes e testa a diferença entre duas médias populacionais, no caso, o grupo de controle e o grupo de teste.

Tabela 2 Teste-t: duas amostras presumindo variâncias equivalentes

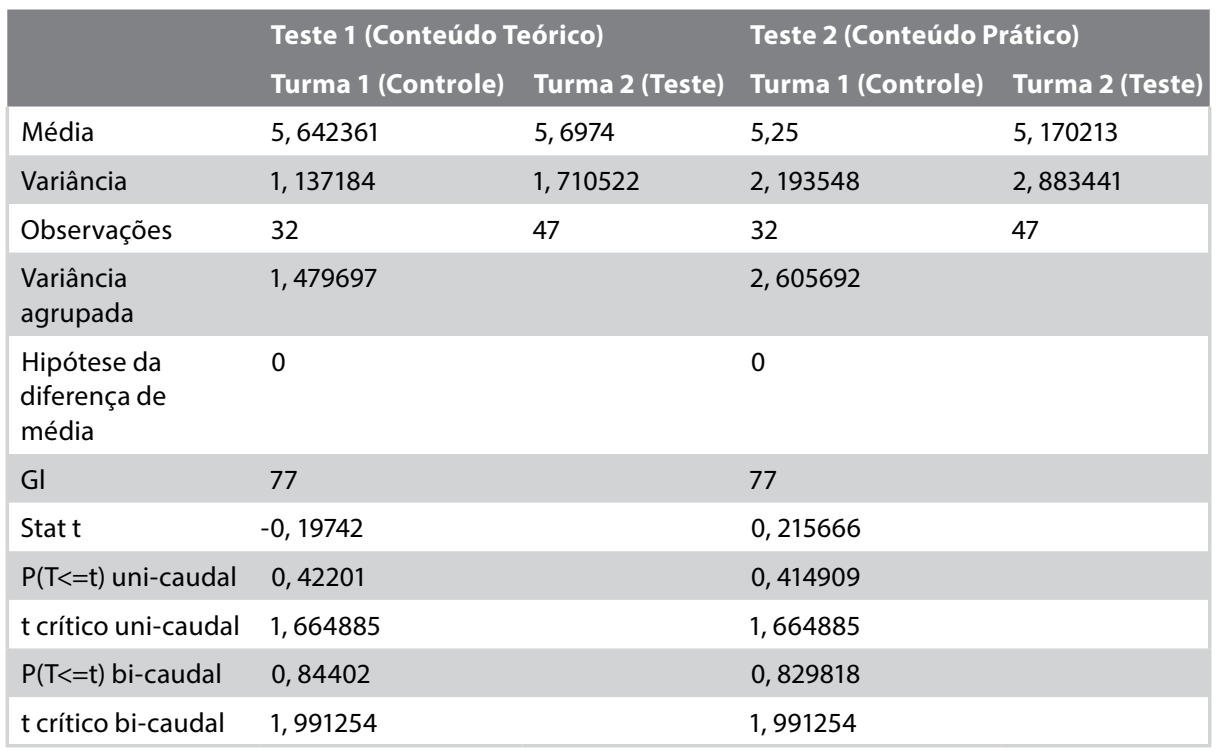

Com base nos resultados do teste " $\mathrm{t}$ ", observa-se que os valores uni-caudal e bi-caudal são altos (distantes de o), demonstrando que as médias são estatisticamente iguais e que o valor calculado "Stat t" é menor que o valor "t crítico uni-caudal" no caso das duas variáveis - Turma i e Turma 2. Desta forma, se aceita a hipótese nula $\left(\mathrm{H}_{\mathrm{o}}\right)$, ou seja, não se pode dizer que existe diferença significativa na absorção do conteúdo ao comparar as médias do teste I (conteúdo teórico) entre os grupos e do teste 2 (conteúdo prático) obtidas pelos alunos participantes do experimento.

Num segundo momento, os mesmos testes foram aplicados na tentativa de encontrar melhorias significativas no aprendizado do teste I (conteúdo 
teórico) para o teste 2 (conteúdo prático - aplicado após a simulação) na mesma turma. Os resultados de ambos os grupos podem ser observados na Tabela 3.

Tabela 3 Teste "F": Teste I - Teste 2

\begin{tabular}{|c|c|c|c|c|}
\hline & \multicolumn{2}{|c|}{ Turma 1 (Grupo de controle) } & \multicolumn{2}{|c|}{ Turma 2 (Grupo de teste) } \\
\hline & $\begin{array}{l}\text { Teste } 1 \\
\text { (Conteúdo } \\
\text { teórico) }\end{array}$ & $\begin{array}{l}\text { Teste } 2 \\
\text { (Conteúdo } \\
\text { prático) }\end{array}$ & $\begin{array}{l}\text { Teste } 1 \\
\text { (Conteúdo } \\
\text { teórico) }\end{array}$ & $\begin{array}{l}\text { Teste } 2 \\
\text { (Conteúdo } \\
\text { prático) }\end{array}$ \\
\hline Média & 0,160373 & 0,963553634 & $-0,05347$ & 0,939626786 \\
\hline Variância & 0,694366 & 0,105697076 & 0,645209 & 0,120679224 \\
\hline Observações & 32 & 32 & 46 & 46 \\
\hline GI & 31 & 31 & 45 & 45 \\
\hline $\mathrm{F}$ & 6,569394 & & 5,346482 & \\
\hline$P(F<=f)$ uni-caudal & $5,33 \mathrm{E}-07$ & & $5,5 \mathrm{E}-08$ & \\
\hline F crítico uni-caudal & 1,822132 & & 1,641516 & \\
\hline
\end{tabular}

Desta forma, observa-se que embora tenha ocorrido melhorias no desempenho do teste I para o teste 2 , isto não pode ser considerado estatisticamente relevante. A aplicação do teste "t " faz-se necessária ao considerar que as variâncias são equivalentes (Tabela 4), porém, como se pode observar tanto os valores uni-caudal quanto bi-caudal são altos, confirmando os testes anteriores que não rejeitam a hipótese nula $\left(\mathrm{H}_{\mathrm{o}}\right)$.

Tabela 4 Teste " $t$ ": Teste $\mathrm{I}$ - Teste 2

\begin{tabular}{|lllll|} 
& \multicolumn{2}{c}{ Turma 1 (Grupo de controle) } & \multicolumn{2}{c|}{ Turma 2 (Grupo de teste) } \\
& Teste 1 & Teste 2 & Teste 1 & Teste 2 \\
\hline Média & 0,160373 & 0,963553634 & $-0,05347$ & 0,939626786 \\
\hline Variância & 0,694366 & 0,105697076 & 0,645209 & 0,120679224 \\
\hline Stat t & $-5,07956$ & & $-7,69637$ & \\
\hline$P(T<=t)$ uni-caudal & $1,86 \mathrm{E}-06$ & & $8,57 \mathrm{E}-12$ & \\
\hline $\mathrm{t}$ crítico uni-caudal & 1,669804 & & 1,661961 & \\
\hline $\mathrm{P}(\mathrm{T}<=\mathrm{t})$ bi-caudal & $3,72 \mathrm{E}-06$ & & $1,71 \mathrm{E}-11$ & \\
\hline $\mathrm{t}$ crítico bi-caudal & 1,998971 & & 1,986674 & \\
\hline
\end{tabular}


Na última etapa realizou-se uma comparação do coeficiente de rendimento (CR) dos alunos participantes do experimento com as médias obtidas em cada teste de conteúdo (teórico e prático) criando um indicador relativo, conforme a Equação I.

(I) $\quad \mathrm{IR}_{\mathrm{x}}=\frac{\mathrm{T}_{\mathrm{Ix}}}{\mathrm{CR}_{\mathrm{x}}}$

Em que: IR corresponde ao Indicador Relativo referente ao aluno $\mathrm{x} ; \mathrm{T}_{\mathrm{Ix}}$ corresponde ao teste de conteúdo do aluno $\mathrm{x}$ e $\mathrm{CR}_{\mathrm{x}}$ ao Coeficiente de Rendimento do aluno x.

Os grupos foram formados por ordem decrescente do coeficiente de rendimento e agrupados em quartis. Os resultados podem ser observados na Tabela 5.

Tabela 5 Indicadores Relativos ao Coeficiente de Rendimento (CR) Grupo de Controle (Turma 1)

\begin{tabular}{|lllllll|}
\hline Grupo & CR & Teste 1 & Indicador & Teste 2 & Indicador & Var (It2/lt1) \\
\hline 1 & 8,25 & 6,1 & 0,74067 & 5,6 & 0,68354 & 0,922867 \\
\hline 2 & 7,255 & 5,7 & 0,78754 & 4,1 & 0,56835 & 0,721678 \\
\hline 3 & 6,5338 & 5,6 & 0,86192 & 6,0 & 0,91928 & 1,066549 \\
\hline 4 & 4,8813 & 5,1 & 1,08013 & 5,3 & 1,11875 & 1,035755 \\
\hline
\end{tabular}

Grupo de Teste (Turma 2)

\begin{tabular}{|lllllll|}
\hline Grupo & CR & Teste 1 & Relativa CR & Teste 2 & Relativa CR & Var (It2/lt1) \\
\hline 1 & 8,098333 & 6,0 & 0,743601 & 6,1 & 0,752105 & 1,011436 \\
\hline 2 & 7,357273 & 6,1 & 0,843386 & 5,5 & 0,752595 & 0,892349 \\
\hline 3 & 6,664167 & 5,7 & 0,846891 & 4,8 & 0,724982 & 0,856051 \\
\hline 4 & 5,04 & 5,0 & 0,969809 & 4,3 & 0,819381 & 0,844889 \\
\hline
\end{tabular}

Observam-se com os resultados de ambas as turmas que os grupos com os maiores coeficientes de rendimento obtiveram as maiores médias no teste I - conteúdo teórico, indicando que o conhecimento acumulado nas demais disciplinas contribuiu para o desempenho superior no teste I, aplicado antes da simulação. 
No desempenho do teste 2 - conteúdo prático, aplicado ao final do experimento, observa-se que os grupos com maiores médias da turma de teste tiveram o mesmo comportamento, ou seja, os grupos com maiores coeficientes de rendimento obtiveram as maiores médias no teste 2. Neste caso, nota-se que a inserção do método do caso na aplicação dos jogos de empresas não provocou alterações na absorção do conhecimento teórico, considerando o que as médias do teste 2 obtiveram o mesmo comportamento do CR.

No entanto, o grupo de controle apresentou um comportamento distinto no que diz respeito ao desempenho do teste de conteúdo prático - teste 2. Os grupos que obtiveram as maiores médias no teste 2 não necessariamente possuem os maiores coeficientes de rendimento, como ocorrido com o Grupo 3 (Tabela 5). Isso mostra que a absorção de conhecimento durante a simulação, atestada no teste de conteúdo prático, não está relacionada ao nível de conhecimento acumulado anteriormente.

Os indicadores relativos mostraram no grupo de controle que os grupos com os menores coeficientes de rendimento obtiveram algum tipo de melhoria da aplicação do teste I para o teste 2. No grupo de teste, apenas um grupo teve seu desempenho melhorado, porém, não foi considerado relevante.

Vale ressaltar que o coeficiente de rendimento analisado no estudo foi obtido após o término do semestre em que ocorreu o experimento, sendo assim, o CR utilizado contém a média da disciplina que ocorre o experimento e as disciplinas cursadas pelos participantes em paralelo. 


\section{CONSIDERAÇÕES FINAIS}

O estudo propôs uma análise das principais vantagens da aplicação conjunta das técnicas de jogos de empresas e do método do caso em comparação à aplicação exclusiva de jogos de empresas. As referidas técnicas oferecem diferentes experiências de aprendizagem e dão ênfase a diferentes habilidades.

A combinação de ambas as técnicas buscou reunir as vantagens e reduzir as limitações e avaliar sua contribuição ao processo de ensino e aprendizagem em administração, uma vez que a melhoria destes processos exige a aplicação de técnicas que insira os alunos em uma participação ativa em análises de casos, aproxime-os da prática da profissão de administrador por meio de vivência, solução de problemas e troca de experiências.

Com a aplicação dos testes estatísticos em todas as etapas concluiu-se que não foram encontradas evidências de melhorias ou perdas significativas no processo de ensino e aprendizagem em administração por meio da aplicação dos jogos de empresas e do método do caso em comparação à aplicação exclusiva de jogos de empresas. Desta forma, a hipótese nula - o quadro teórico apresentado da turma de teste não apresenta perda ou ganho na absorção do conhecimento em relação à turma de controle, mesmo sendo expostas a duas abordagens diferentes - não foi rejeitada.

Os testes $\mathrm{F}$ e " $\mathrm{t}$ " de student foram aplicados com o uso de variáveis distintas, no entanto, concluiu-se que não há diferenças consideradas relevantes estatisticamente: (I) entre as médias de nenhum dos testes de conteúdo entre nenhuma das turmas - teste e controle; (2) do resultado do teste I para o teste $2 \mathrm{em}$ ambas as turmas.

Conclui-se então, que embora a literatura seja favorável a aplicação do método do caso com os jogos de empresas, neste estudo, não foram encontradas evidências de melhorias ou perdas na assimilação do conhecimento, porém, não se pode concluir que a aplicação das técnicas em conjunto não apresenta vantagens e não contribui para a melhoria do processo de ensino e aprendizagem em administração. 
Neste estudo, os resultados apontaram que embora o método do caso proporcione o desenvolvimento de conhecimento (MOTTA; ARMOND-DE-MELO, 2008), sua utilização para apresentação das regras econômicas do simulador, etapa distintiva entre os grupos estabelecidos na pesquisa, não apresentou ganhos na assimilação dos conteúdos programáticos da disciplina. Da mesma forma, os resultados não apresentaram perdas na assimilação dos conteúdos programáticos.

Os resultados desta investigação contribuíram para o avanço do conhecimento na área de ensino e aprendizagem em administração, possibilitando a combinação de duas técnicas que estão se tornando populares nos cursos de administração, além de oportunizar reflexões críticas sobre o processo.

As principais dificuldades encontradas estão associadas à aplicação do experimento. Primeiramente, a quantidade de alunos expostos à experiência, ou seja, o tamanho da amostra, dificultando a generalização do estudo. Outra limitação se dá ao fato de uma turma ser integral e outra noturna, podendo alguns alunos ter tido mais tempo disponível para dedicar-se a disciplina e outros não. Além do fato do teste de conteúdo prático ter sido aplicado ao final do período quando há maior número de atividades acadêmicas.

Os casos de ensino desenvolvidos para aplicação do experimento podem não ter sido adequados, sendo estes muito extensos ou com poucos exemplos práticos dificultando a assimilação do conhecimento. E por fim, não se considerou diferenças de nível de dificuldade entre os testes aplicados, pois o segundo teste possuía um nível mais avançado, assim, se considerado um peso maior os resultados poderiam evidenciar melhorias nos resultados.

É importante ressaltar que o estudo não é conclusivo e exige um esforço ainda maior por parte dos pesquisadores na replicação do experimento, revisão dos casos empresariais desenvolvidos e dos exercícios, aplicação em outras instituições e levantamento de opiniões dos participantes sobre as cinco variáveis apresentadas por Raia (1966). Sugere-se a reaplicação do experimento em outras turmas para ampliar o tamanho da amostra; o tratamento dos dados com o uso de outras técnicas estatísticas, como por 
exemplo, análise multivariada de variâncias (MANOva) e análise de associação não linear entre as variáveis do estudo e a aplicação de inventários antes do início do experimento para identificar os estilos de aprendizagem, como por exemplo, o Index Learning Styles - ILs de Felder-Saloman. 


\section{REFERÊNCIAS}

ARBEX, M.A. O valor pedagógico dos jogos de empresas na aprendizagem de gestão de negócios. Revista FAE, v. 8, n. 2, p. 81-89, 2005.

BARNES, L. B.; CHRISTENSEN, C. R.; HANSEN, A. J. Teaching and the Case Method. Boston, Massachusetts: Havard Business School Press, 1994.

BENNIS, W.; O’TOOLE, J. How business schools lost their way. Harvard Business Review, v. 85, n. 5, p. 96-104, 2005.

BERTERO, C. O. Ensino e pesquisa em administração. São Paulo: Thomson Learning, 2006.

. A problemática educação de administradores. Revista de Administração

Eletrônica, v. 43, n. 2, p.10-25, 2003.

BERTERO, C. O.; CALDAS, M. P., WOOD JR., T. Produção científica em administração de

empresas: provocações, insinuações e contribuições para um debate local. Revista de Administração Contemporânea, v. 3, n. 1, p. 147-178, 1999.

BORDENAVE, J. D.; PEREIRA, A. M. Estratégias de ensino-aprendizagem. Petropolis, RJ: Vozes, 2006.

DICKINSON J.R.; FARIA, A.J. The teaching effectiveness of games in collegiate business courses. Simulation and Games, v. 4, n. 3, p. 259-294, 1994.

IKEDA, A. A.; VELUDO-DE-OLIVEIRA, T.; CAMPOMAR, M. C. A Tipologia do método do caso em Administração: usos e aplicações. Organizações e Sociedade, v.12, n. 34, p.141-159, 2005.

INEP - Instituto Nacional de Estudos e Pesquisas Educacionais Anísio Teixeira (2009). Resumo Técnico - Censo da Educação Superior de 2009. Brasília - DF, 2010. Disponível em: http://download.inep.gov.br/download/superior/censo/2009/resumo tecnico2009. pdf. Acesso em: 12/09/2011.

KATZ, R.L. Skills of an Effective Administrator. Harvard Business Review, v. 54, n. 94, 1974.

KEYS, B.; WOLF. J. The role of management games and simulations in education and research. Journal of management, v. 16, n. 2, p. 307-336, 1990.

KOLB, David. A. Experimental Learning: experience as a source of learning and development. New Jersey: Prentice Hall, 1984.

LI, E.; BAILLIE, A. Mixing case method with business game: student evaluations. Simulation \& Gaming, v. 24, n. 3, p. 336-355, 1993.

MENEZES, M.A. de A. Do método do caso ao case: a trajetória de uma ferramenta pedagógica. Educação e Pesquisa, v.35, n.1, p. 129-143, 2009. 
MINTZBERG, H. Managers not MBAs: A hard look at the soft practice of managing and management development. San Francisco: Berrett Koehler, 2004.

MINZTBERG, H.; GOSLING, J. Educando administradores além das fronteiras. Revista de Administração de Empresas, v. 43, n. 2, p. 29-43, 2003.

MOTTA, G.; ARMOND-DE-MELO, D. R. A prática da aprendizagem baseada em problemas nos cursos de gestão. In: Colóquio Internacional sobre Ensino Superior, Feira de Santana, Bahia, Brasil, 1., 2008.

NAKANO, K.; TERANO, T. From Gaming Simulation to Case Method Empirical Study on Business Game - Development and Evaluation. In: International Conference, 8th, KES 2004, Wellington, New Zealand, p. 472-479, 2004.

A Case-Oriented Game for Business Learning. Dept. Computational Intelligence and Systems Sciences, Tokyo Institute of Technology, Tokyo, p. 779-784, 2005.

NAKANO, K.; MATSUYAMA, S.; TERANO, T. Research on a Learning System toward Integration of Case Method and Business Gaming. Tokyo Institute of Technology, Tokyo, 2007.

NICOLINI, A. Qual será o futuro da fábrica de administradores? Revista de Administração Eletrônica, v. 43, n. 2, p. 44-54, 2003.

OLIVEIRA, J.F. de. Cases: os mais famosos estudos de casos internacionais... São Paulo: Érica, 2000.

OLIVEIRA, M. A.; SAUAIA, A. C. A. Impressão Docente para Aprendizagem Vivencial: Um Estudo dos Benefícios dos Jogos de Empresas. Administração: Ensino e Pesquisa, v. 12, n. 3, p. 355-391, 2011.

PFEFFER, J.; FONG, C. O fim das escolas de negócios? Revista de Administração Eletrônica, v. 43, n. 2, p. 11-28, 2003.

PRETTO, F.; FILARDI, F. Jogos de empresas: Uma estratégia de motivação no processo de ensino e aprendizagem dos cursos de Administração. Globadvantage - Center of Research in International Business \& Strategy. Working Paper, 13., 2008.

PRETTO, F. N.; ALMEIDA, F. C. Proposta de um novo modelo de Jogos de Empresas. In: EnAnpad, 31., 2007, Rio de Janeiro. Anais...Rio de Janeiro: Anpad, 2007.

RAIA, A. A study of the educational value of the management games. Journal of Business, v. 39, n. 3, p. 339-352, 1966.

ROESCH, S.M.A. Casos de ensino em administração. Revista de Administração Contemporânea, v. 11, n. 2, p. 213-234, 2007.

ROESCH, S. M.; FERNANDES, F. Construção de Casos para Ensino. Revista ANGRAD, v. 7, n. 4, 2006.

ROSAS, A. R.; SAUAIA, A. C. A. Modelo Conceitual de Decisões no Estágio de Criação de um Negócio: Base para Construção de um Simulador para Jogos de Empresas.

Revista de Administração Contemporânea, v. 13, n. 4, p. 663-682, 2009. 
Jogos de Empresas na Educação Superior no Brasil: Perspectivas para 2010. In: EnAnpad, 30., 2006, Salvador. Anais... Salvador: Anpad, 2006.

SANTOS, R. V. dos. Jogos de empresas aplicados ao processo de ensino e aprendizagem de contabilidade. Revista de Contabilidade e Finanças, n. 31, p. 78 - 95, 2003.

SANTOS, M. R. G. F.; LOVATO, S. Os Jogos de Empresas como Recurso Didático na Formação de Administradores. In: Ciclo de Palestras Novas Tecnologias da Educação CINTED-UFRGS, v. 5, n. 2, 2007.

SAUAIA, A.C.A. Cases and business games: the perfect match! Developments in Business Simulation and Experiential Learning, v. 33, n.1, p. 204-212, 2006.

. Laboratório de Gestão: simulador organizacional, jogo de empresas e pesquisa aplicada. 2.ed. Barueri: Manole, 2010.

SILVA, S. S.; OLIVEIRA, M. A. MOTTA, G. S. Estudo combinado entre Jogos de Empresas e o Método do Caso: Avaliação da Contribuição ao Processo de Ensino e Aprendizagem em Administração. In: Semead, 15., 2012, São Paulo. Anais... São Paulo: USP, 2012.

STAHL, L. M.; LOPES, P. C. Estratégias de avaliação para jogos de empresas gerais: avaliando desempenho ou aprendizagem. In: EnAnpad, 34., Curitiba, 2004. Anais... Curitiba: Anpad, 2004.

TOMPSON, G. H.; DASS, P. Improving students' self-efficacy in strategic management: The relative impact of cases and simulations. Simulation \& Gaming, v. 31, n. 22, 2000.

TUSHMAN, M.; O’REILLY, C.; FENOLLOSA, A.; KLEINBAUM, A.; McGRATH, D. Relevance and rigor: executive education a lever in shaping practice and research. Academy of Management Learning \& Education, v. 6, n. 3, p. 345-362, 2007. 


\section{DADOS DOS AUTORES}

SHEILA SERAFIM DA SILVA`sheila_serafim@yahoo.com.br Graduação em Administração pela Universidade Federal Fluminense Instituição de vinculação: Universidade Federal Fluminense Volta Redonda/RJ - Brasil

Áreas de interesse em pesquisa: Ensino e Aprendizagem em Administração; Jogos de empresas e Simuladores Organizacionais.

* Rua Desembargador Ellis Hermydio Figueira, 783

Aterrado Volta Redonda/RJ 27213-145

MURILO ALVARENGA OLIVEIRA malvarenga@id.uff.br

Doutor em Administração pela Universidade de São Paulo

Instituição de vinculação: Universidade Federal Fluminense

Volta Redonda/RJ - Brasil

Áreas de interesse em pesquisa: Apoio a Decisão e Avaliação de Desempenho

Organizacional; Jogos de Empresas, Simuladores Organizacionais e Metodologia

Vivencial; Estratégia, Tecnologia e Inovação.

GUSTAVO DA SILVA MOTTA gustavosmotta@gmail.com

Doutor em Administração pela Universidade Federal da Bahia

Instituição de vinculação: Universidade Federal Fluminense

Volta Redonda/RJ - Brasil

Áreas de interesse em pesquisa: Jogos de Empresas; Ciência, Tecnologia e Inovação. 